$\mathrm{T}$ he treatment strategy for acute myocardial infarction (MI) with ST elevation or newly developed left bundle branch block has been focusing on immediate opening of the infarct related coronary artery. This is because the prognosis for the patient is dependent upon the restoration of coronary flow and myocardial perfusion. ${ }^{1}$ Numerous randomised controlled trials with thrombolytic drugs have shown that these drugs can preserve left ventricular function and decrease mortality. Therefore treatment with streptokinase, alteplase, reteplase, and tenecteplase, when administered within 12 hours of onset of symptoms, is given the highest recommendation (IA) in guidelines. ${ }^{2}$

\section{PERCUTANEOUS CORONARY INTERVENTION}

Mechanical reperfusion with percutaneous coronary intervention (PCI) in acute MI (primary or direct PCI) (fig 1) was first performed by Meyer and colleagues and Hartzler and colleagues in the beginning of the 1980s. During the next decade pioneer work, in particular by the Zwolle and the PAMI groups, showed that when performed in centres with great expertise, primary PCI was superior to thrombolytic treatment. Subsequent trials conducted in centres, that were not necessarily staffed by world experts in primary PCI, also showed that in this setting (the real world) primary PCI was better than thrombolytic treatment. In a recent meta-analysis of 23 randomised trials comparing primary PCI and thrombolytic treatment, primary PCI was superior to thrombolysis, when looking at short term mortality (7\% v9\%), non-fatal reinfarction (3\% $v$ $7 \%)$, stroke ( $1 \% \vee 2 \%)$, and the combined end point of death, non-fatal re-infarction, and stroke. ${ }^{3}$

In 1999 the American College of Cardiology/American Heart Association guidelines recommended primary PCI as an alternative to thrombolysis. The European guidelines published in $2003^{2}$ state: "primary PCI is the preferred therapeutic option when it can be performed within 90 minutes after the first medical contact. It requires an experienced team, which includes not only interventional cardiologists, but also skilled supporting staff. This means that only hospitals with an established interventional cardiology programme should use primary PCI as a routine treatment option."

Guidelines are the result of a consensus of leading experts based upon a thorough review of the existing scientific literature. The European guidelines were accepted for publication in August 2002. After this publication various improvements in reperfusion therapy, both to refine thrombolytic treatment and primary PCI, have been assessed in several recent trials. Particular focus has been put on the possibility of transferring patients with acute ST elevation MI from district hospitals to tertiary units with PCI facilities for primary PCI.

\section{TRANSFERRAL FOR PRIMARY PCI?}

Because of geographical reasons and lack of staff, including experienced PCI operators, establishment of 24 hour facilities for primary PCI may not be available in all hospitals, where patients with acute MI are admitted. Therefore thrombolytic therapy is still the prevailing treatment in most countries.

For patients with a relative short distance to travel from the district hospital of admission to a tertiary centre with a 24 primary PCI service, immediate transferral might be an option. The strategies of either administering thrombolytic treatment in the hospital of admission, or transferring the patient (without giving thrombolytic treatment) to a PCI centre, have recently been tested in five randomised studies.

The Maastricht trial ${ }^{4}$ randomised 224 patients with acute ST elevation MI and symptoms of $<6$ hours duration to treatment with alteplase, alteplase, and transferral for treatment with PCI (if necessary), or immediate transferral for primary PCI. This pioneering trial was a feasibility and safety study, which showed that transferral was safe. There was a trend towards decreased mortality and recurrent MI in the primary PCI group. 

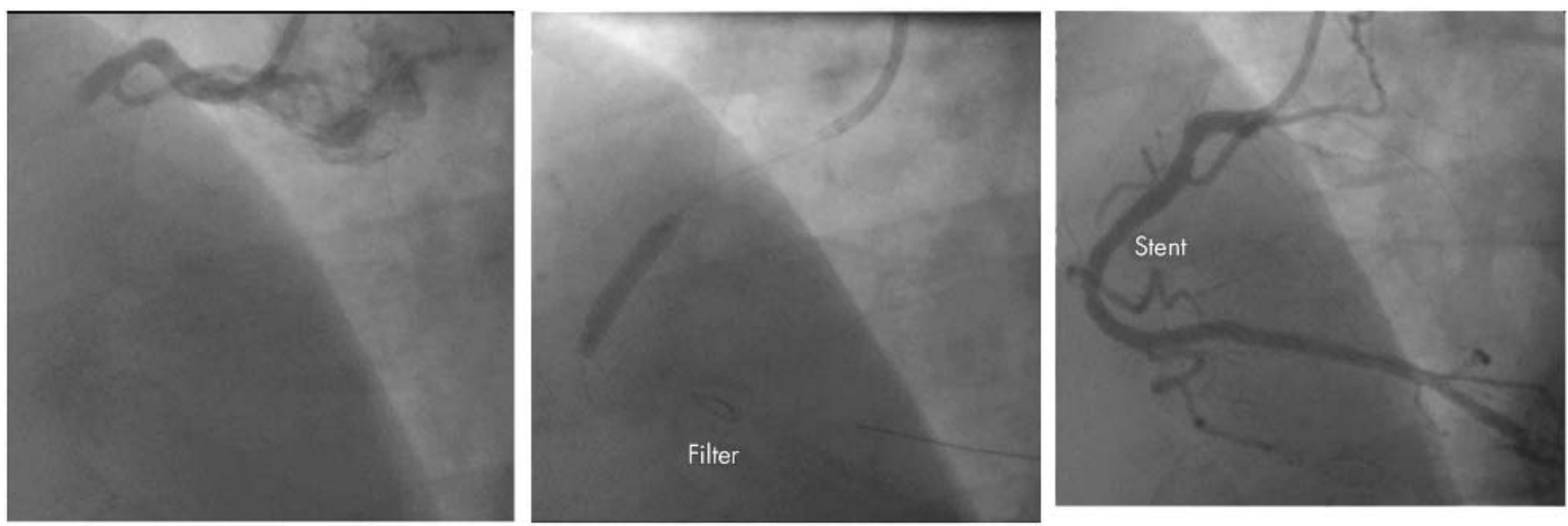

Figure 1 Inferior ST elevation myocardial infarction with total occlusion of the right coronary artery treated with a stent and a distal protection device.

The PRAGUE trial ${ }^{5}$ randomised 300 patients with ST elevation MI and symptoms of $<6$ hours duration to treatment with streptokinase, treatment with streptokinase and transferral for PCI, or transferral for primary PCI. Transfer was safe and transferral for primary PCI was associated with a significantly lower incidence of the composite end point of death/reinfarction/stroke at 30 days compared to the other treatment groups. This difference was mainly due to a very low incidence of reinfarction in the primary PCI group.

AIRPAMI $^{6}$ randomised 138 high risk, acute ST elevation infarct patients with symptoms of $<12$ hours duration to tissue plasminogen activator (t-PA) (plus 48 hours of unfractionated heparin infusion) in the local hospital or transferral for primary PCI. The primary end point was the combination of death/reinfarction/disabling stroke within 30 days. The trial was stopped prematurely because of difficulties in recruiting patients. The transfer group had a $38 \%$ reduction in the primary end point, but this difference was not significant. The time to treatment was delayed in the primary PCI group, mainly because of a delay in the initiation of transport.

PRAGUE- $2^{7}$ was a multicentre trial performed in the Czech Republic. Eight hundred and fifty patients with acute ST elevation MI presenting within 12 hours of symptoms admitted to the nearest community hospital without PCI facilities were randomised to either thrombolysis (streptokinase) in this hospital or immediate transport for primary PCI. The primary end point (30 day mortality) occurred in $6.8 \%$ in the PCI group compared to $10.0 \%$ in the streptokinase group $(p=0.12)$. Among 299 patients who were randomised with $>3$ hours of symptoms the mortality rate for primary PCI was $6 \% \vee 15.3 \%$ in the thrombolysis group $(\mathrm{p}<0.02)$. For patients randomised with $<3$ hours of symptoms $(\mathrm{n}=551)$ there was no difference in mortality between the primary PCI group $(7.3 \%)$ and the thrombolysis group (7.4\%).

DANAMI- $2^{8}$ was a Danish randomised, multicentre, controlled trial that assigned 1572 patients to alteplase (plus 48 hours of unfractionated heparin) or primary PCI. The primary end point was a combination of death/clinical reinfarction/ disabling stroke at 30 days. $^{89}$ A total of 1129 patients were enrolled at referral hospitals and 443 patients were randomised in centres with PCI facilities. Among patients who underwent randomisation at referral centres the primary end point occurred in $8.5 \%$ of the patients transferred for PCI and in $14.2 \%$ of the patients treated with alteplase $(p=0.002)$.
In patients randomised in the hospitals with PCI facilities, $6.7 \%$ in the PCI group reached the primary end point compared to $12.3 \%$ in the thrombolysis group $(p=0.05)$. Among all patients the better outcome after primary PCI was driven by a reduction in reinfarction $(1.6 \%$ in the primary PCI $v 6.3 \%$ in the thrombolysis group; $\mathrm{p}<0.001)$; no significant differences were observed in the rate of death $(6.6 \% \vee 7.8 \%$; $\mathrm{p}=0.35)$ or the rate of stroke $(1.1 \% \vee 2.0 \% ; \mathrm{p}=0.15)$. The superiority of primary PCI over thrombolysis was present in patients randomised within $<2$ hours of symptom onset, those randomised between 2-4 hours of symptom onset, and in patients with symptoms of $\geqslant 4$ hours duration.

The data from the trials that have compared transfer to primary PCI with thrombolysis have recently been analysed in two meta-analyses. Keeley and colleagues ${ }^{3}$ did a quantitative review of 23 trials on primary PCI versus thrombolysis and also a separate meta-analysis of the five trials described above. This meta-analysis on the transfer trials $(n=2909)$ showed that despite the delay in receiving reperfusion therapy inherent in transfer (average 39 minutes), primary PCI was associated with significant reductions in non-fatal reinfarction, total stroke, and the combined end point of death, non-fatal reinfarction, or stroke at 30 days. Transfer for primary PCI was non-significantly associated with a decrease in death rate compared with thrombolytic treatment ( $\mathrm{p}=0.057)$.

In another meta-analysis, Dalby and colleagues ${ }^{10}$ analysed the data from the five trials mentioned above plus the CAPTIM study ${ }^{11}$ described below $(\mathrm{n}=3750)$. The combined end point of death/reinfarction/stroke was significantly

\section{Trial acronyms}

AIRPAMI: Air Primary Angioplasty in Myocardial Infarction

- BRAVE: Bavarian Reperfusion Alternatives Evaluation

- CAPTIM: Comparison of Angioplasty and Pre-hospital Thrombolysis in acute Myocardial infarction

DANAMI: Danish trial in Acute Myocardial Infarction

PACT: Plasminogen-activator Angioplasty Compatibility Trial

- PRAGUE: Primary angioplasty in patients transferred from general community hospitals to specialized PTCA units with or without emergency thrombolysis

- SHOCK: Should we emergently revascularize Occluded Coronaries for cardiogenic shock? 
reduced by $42 \%$ in the group transferred for primary PCI compared to the group that received on-site thrombolysis. When looking at the end points separately, reinfarction was significantly reduced by $68 \%$ and stroke by $56 \%$. There was a trend towards a reduction of all cause mortality of $19 \%$ at 30 days $(\mathrm{p}=0.08)$.

1360 At the present time no single study has shown that transfer for primary PCI decreases mortality significantly compared to thrombolytic treatment. The only trial designed to show this was the PRAGUE-2 trial, and although 30 day mortality was reduced by $32 \%$, this did not reach significance. ${ }^{7}$ The DANAMI-2 trial convincingly showed that transfer for primary PCI was associated with a better outcome, with the number needed to treat to prevent one incidence of death/ clinical re-infarction/disabling stroke being $18 .^{8}$ This difference was mainly driven by a substantial reduction in reinfarction, that was judged by an end point committee using very strict criteria for definition of reinfarction. ${ }^{8}$ DANAMI-2 was not powered to show changes in mortality, and the smaller PRAGUE-2 might have been underpowered to show changes in mortality.

In conclusion, transferral for PCI has been shown to be superior to thrombolysis. This is mainly caused by a substantial reduction in reinfarctions and to a lesser extent by a reduction in stroke and death at 30 days. Long term follow up of the trials is warranted.

\section{CAN ALL PATIENTS BE TRANSFERRED SAFELY?}

In the Maastricht and PRAGUE trials transfer was found to be safe. ${ }^{457}$ In the Maastricht trial the patients were transported in ambulances with well trained paramedic staff. ${ }^{4}$ In the AIRPAMI trial ${ }^{6}$ transfer was done by air or ground transportation and was found to be safe, despite the inclusion of high risk patients.

Ninety six per cent of the patients in DANAMI-2 randomised to transfer for PCI were transferred from referral hospitals to an invasive PCI centre within two hours. ${ }^{8}$ Of the 567 patients randomly assigned to transfer to primary PCI at the referral hospital, 559 patients were actually transferred (99\%). No deaths occurred during transport. Eight patients had ventricular fibrillation and one of the patients had refractory ventricular fibrillation on arrival at the PCI centre and died one hour later after unsuccessful resuscitation. In the PRAGUE-2 trial ${ }^{7}$ two patients died and three were successfully treated with DC conversion for ventricular fibrillation during transport.

When merging all safety data from the five trials, Keeley and colleagues ${ }^{3}$ reported that transfer was associated with a $0.5 \%$ risk of death (reported in the PRAGUE- 2 trial only), and that there was a $0.7-1.4 \%$ risk of ventricular arrhythmias and a $2 \%$ risk of second degree or third degree heart block.

It is obvious that the criteria for selection of patients for transfer are very important. In the DANAMI- 2 trial $4 \%$ of the patients screened at the referral hospitals were judged to be unable to tolerate transportation. In most of the trials patients with cardiogenic shock were excluded because it was felt that these patients should be treated with primary PCI based on evidence from the SHOCK trial. ${ }^{12}$

In conclusion, transportation of patients with acute ST elevation MI was found to be safe. In the DANAMI-2 trial patients were transported in ambulances with a doctor (trained in cardiac resuscitation), and in some cases also by a nurse with expertise in emergency medicine and anaesthesiology. The rest of the crew comprised two drivers with some experience in treatment of cardiac arrest. In the CAPTIM trial the ambulances were staffed with anaesthetists, who also carried out the pre-hospital randomisation.

\section{SHOULD PATIENTS WITH A SHORT DURATION OF SYMPTOMS BE TRANSFERRED?}

Data from registers and controlled trials show that thrombolysis is an excellent treatment when administered within the first two hours of symptom onset in patients with ST elevation MI. ${ }^{1}$ Thrombolysis is less effective as time passes, whereas primary PCI seems to be very effective after four hours and appears to be less time dependent than thrombolytic treatment. ${ }^{13}{ }^{14}$ In the PRAGUE-2 trial $^{7}$ there was no difference between streptokinase and transferral for primary PCI in patients with symptoms of $<3$ hours duration. On the other hand, in the DANAMI trial ${ }^{8}$ the superiority of transferral for primary PCI was also present in patients presenting early after onset of symptoms. In the DANAMI-2 trial $58 \%$ of the patients were randomised within two hours of symptom onset and $82 \%$ within four hours, so this trial provides evidence that transferral for primary PCI is also a good option in patients presenting early. ${ }^{8}$

\section{IS PRE-HOSPITAL THROMBOLYSIS BETTER THAN IN- HOSPITAL THROMBOLYSIS?}

Clearly, rapid reperfusion by immediate administration of thrombolytics in the ambulance after the diagnosis is established is an attractive option. A meta-analysis of six trials with a total of 6434 patients randomised to pre-hospital or in-hospital thrombolysis revealed a significantly reduced mortality in the pre-hospital thrombolysis group. The estimated time to treatment was 104 minutes in the prehospital group compared to 162 minutes in the in-hospital group. ${ }^{15}$ Clearly, pre-hospital logistics are key in shortening the time to reperfusion therapy and prognosis (fig 2).

\section{IS TRANSFER FOR PRIMARY PCI BETTER THAN PRE- HOSPITAL THROMBOLYSIS?}

This interesting question has been addressed in a French multicentre trial (CAPTIM). ${ }^{10}$ Eight hundred and forty patients with acute ST elevation MI of $<6$ hours duration were randomised in the ambulance to pre-hospital thrombolysis with alteplase or to primary PCI. All patients were admitted to a hospital with acute PCI facilities. The primary end point was the combination of death/reinfarction/nonfatal disabling stroke at 30 days. Patients randomised to prehospital thrombolysis with signs of no reperfusion were treated with rescue PCI at the time of admission (26\%). The primary end point occurred in $8.2 \%$ in the pre-hospital fibrinolysis group and $6.2 \%$ in the primary PCI group. This trend towards a better outcome for primary PCI was not significant. The death rate was $3.8 \%$ in the pre-hospital fibrinolysis group and $4.8 \%$ in the primary angioplasty group (not significant).

Although the study was terminated prematurely because of problems in funding (it was planned to include 1200 patients) and therefore is underpowered, the trial provides interesting information. Further analysis of the data looking at the subgroup of patients randomised within two hours of onset of symptoms $(n=460)$ revealed a strong trend towards a lower 30 day mortality in the pre-hospital fibrinolysis group $(2.2 \%)$ compared to the primary PCI group $(5.7 \%)(\mathrm{p}=0.058) \cdot{ }^{16}$ 

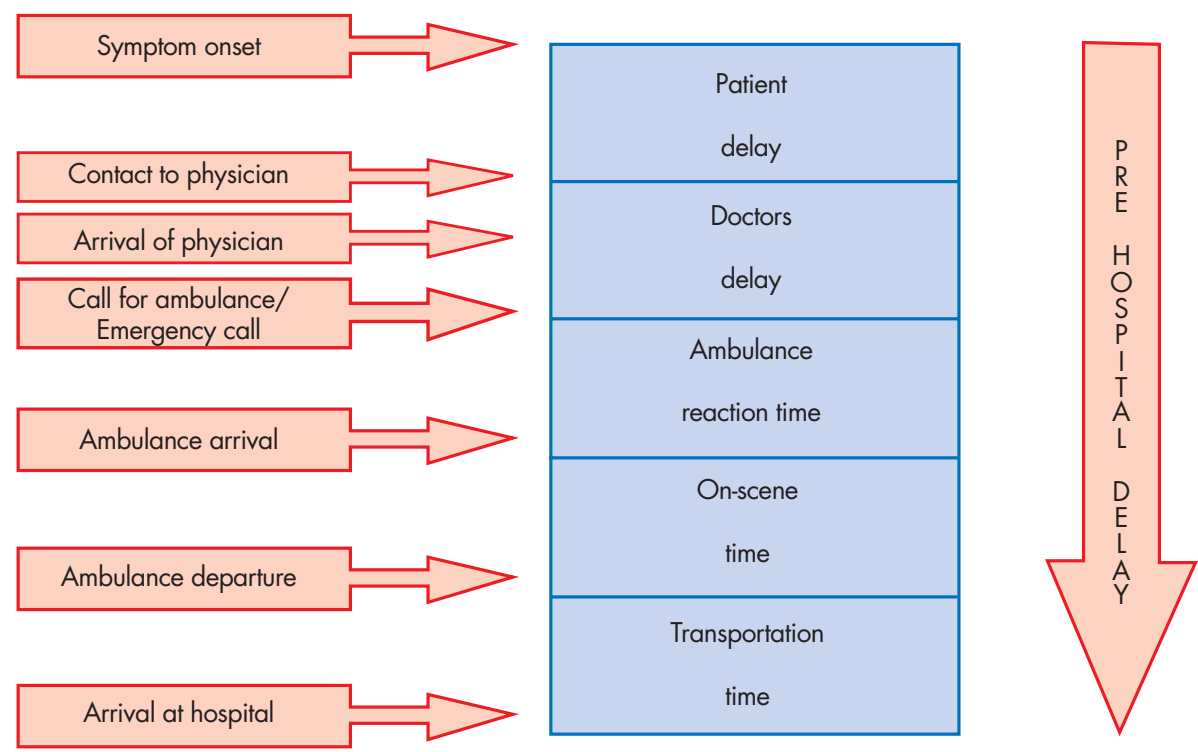

Figure 2 Pre-hospital delay to reperfusion therapy: the key to improving treatment of patients with ST elevation myocardial infarction.

\section{ARE THROMBOLYTIC REGIMENS USED IN STUDIES UP TO DATE?}

Alteplase is generally accepted as being marginally better than streptokinase, particularly in patients presenting with symptoms of $>4$ hours duration. ${ }^{2}$ The double bolus drug, reteplase, and the single bolus drug, tenecteplase, are both considered to be as effective and safe as alteplase, and are now preferred by many clinicians, because they are easier to administer. Although neither reteplase nor tenecteplase have been shown to be superior to alteplase in in-hospital trials, these new drugs are of great potential interest in the prehospital setting.

Much effort has been used to try to optimise adjunctive treatment. The combinations of both thrombolytics and glycoprotein IIb/IIIa inhibitors, and thrombolytics and low molecular weight heparin, have been shown to be more effective anti-thrombotic regimens than the conventional treatment with alteplase and intravenous unfractionated heparin infusion, but trials have failed to show a reduction in mortality. ${ }^{1}$ Also both these combinations are associated with the occurrence of more bleeding episodes. Although studies at the present time are testing the optimal dose of low molecular heparin in combination with bolus thrombolytics, most experts would at the moment still consider alteplase, reteplase, or tenecteplase followed by 48 hours of unfractionated heparin infusion to be the best thrombolytic regimen.

Therefore the thrombolysis arms in the Maastricht study, the AIRPAMI, the DANAMI-2, and the CAPTIM trial can still be considered to be up to date, whereas some experts would criticise the PRAGUE studies for having used streptokinaseparticularly when used in patients with symptoms $>4$ hours duration. $^{2}$

\section{HAVE PCI TECHNIQUE AND ADJUNCTIVE THERAPY IMPROVED AFTER THE TRIALS HAVE BEEN TERMINATED?}

Several recent trials have compared the use of stents with balloon angioplasty in patients undergoing primary PCI, and the use of stents is now recommended in these patients. In the DANAMI-2 trial the use of stents was highly encouraged and was used in $93 \%$ of the PCI patients.

Another recent advance is the use of the platelet glycoprotein IIb/IIIa inhibitor, abciximab, before or during the procedure in the catheterisation laboratory. ${ }^{17}$ In the trials comparing transfer to primary PCI with thrombolysis, abciximab was not used routinely. In the DANAMI-2 trial $39 \%$ of the patients randomised to primary PCI were treated with abciximab.
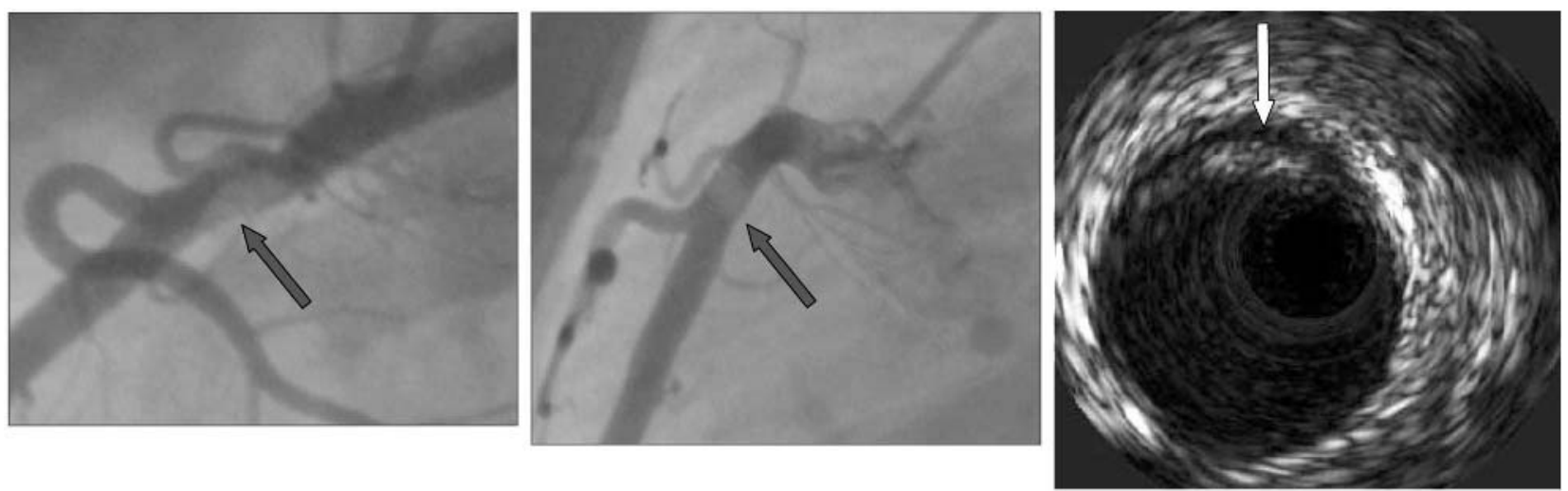

Figure 3 Plaque rupture in the right coronary artery in a 22 year old man presenting with acute ST elevation myocardial infarction. Intravascular ultrasound shows a local fissure at the site of the thrombus. 


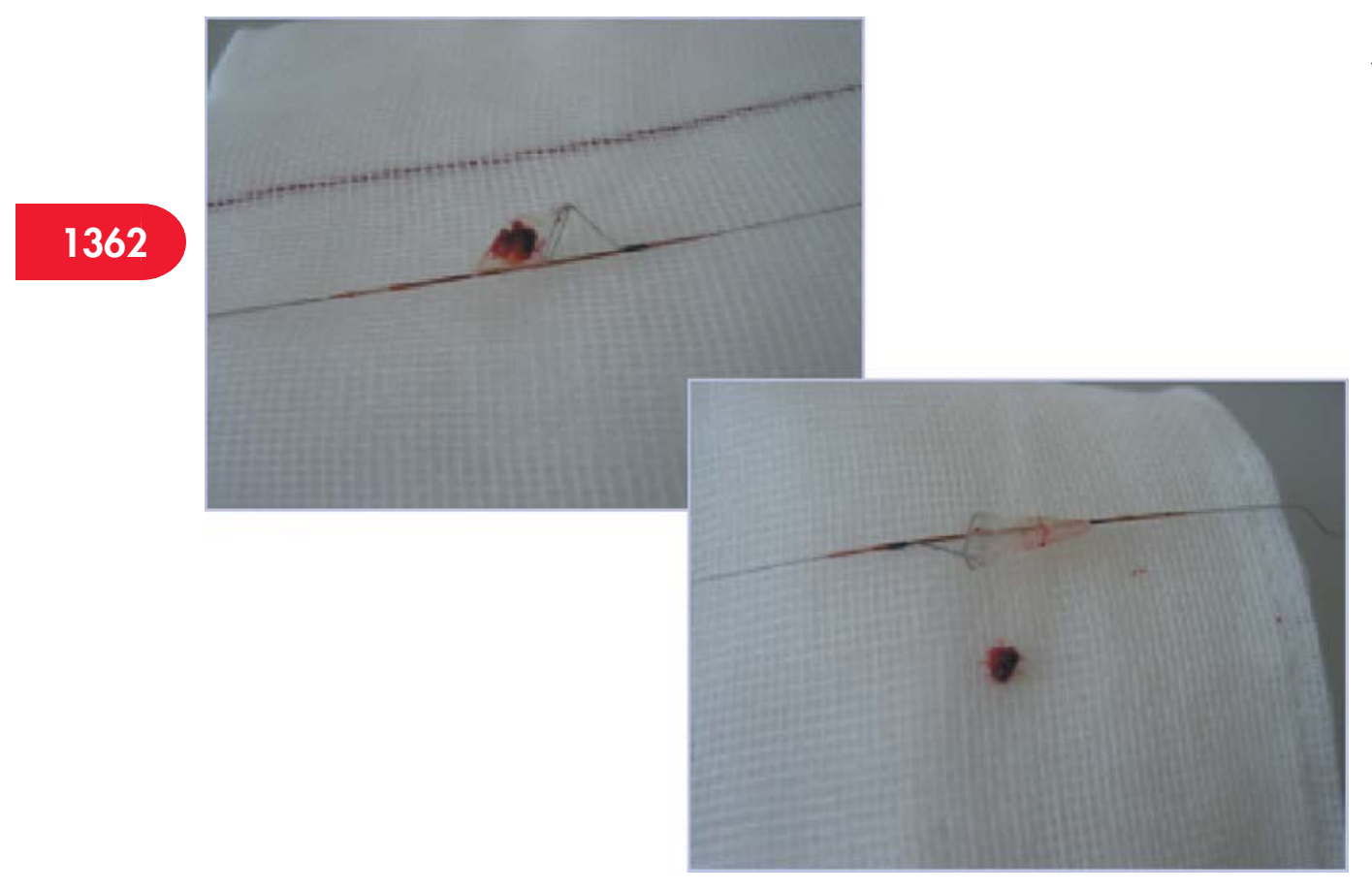

Figure 4 Coronary thrombus caught with a distal protection device during primary $\mathrm{PCl}$.

Recent studies have indicated that quick restoration of flow in the infarct related coronary artery is not the only predictor of outcome: myocardial perfusion as reflected by resolution of ST segment elevation in the ECG, myocardial blush rate assessed during angiography, and myocardial flow measured by scintigraphy are also important prognostic indicators. Embolisation of the coronary thrombus (fig 3) or of material from the atherosclerotic plaque released during PCI may impair the distal coronary circulation and myocardial perfusion; distal mechanical protection devices (figs 1 and 4) and suction (thrombectomy) catheters are currently being tested in a randomised trial on patients undergoing primary PCI, and may further improve outcome in these patients.

In-stent restenosis occurs with a similar or slightly increased rate in primary PCI compared to elective PCI. Introduction of drug eluting stents has significantly decreased restenosis rates in elective PCI and may improve long term outcome also after primary PCI. This is currently being assessed in registries. Other new therapeutic options include inhibition of the inflammatory system by antibodies against the complement system and repair of the infarcted area by autologous stem cells.

It is not clear whether primary PCI technique has improved or whether patient outcome has changed compared to the published randomised trials, but the PCI technique carries a potential for significant improvements.

\section{PRE-HOSPITAL DIAGNOSIS AND TREATMENT}

All available data support the concept that the key to improvement of prognosis in ST elevation MI is to initiate reperfusion therapy as soon as possible after onset of symptoms (fig 2). Early diagnosis on site is a key factor. This can be provided by ambulances staffed with doctors, nurses, or specially trained paramedics that can record and interpret 12 lead ECGs, make a quick and correct diagnosis, initiate treatment with aspirin, heparin, and $\beta$ blockers, and administer pre-hospital thrombolysis or transfer the patient safely to a centre where primary PCI can be performed. If the patient is taken for primary PCI, 300-600 mg of clopidogrel and, if possible, optimal blockade of the platelets with abciximab should be considered in the ambulance. Ideally the patient should go directly to the catheterisation laboratory, where the PCI team is ready to perform angioplasty straight away.

If, for logistic reasons, a system enabling diagnosis and treatment in the ambulance cannot be established, telemedicine offers the possibility to send ECGs electronically to the hospital, where the diagnosis can be made, and the ambulance can be directed to the catheterisation laboratory in the invasive centre, where the PCI crew will be ready (see box). The fact that the PCI team gets this early warning reduces in-hospital delay considerably and facilitates urgent reperfusion with PCI.

\section{THE FUTURE: FACILITATED PCI?}

Defining the optimal standard of care may not be a choice between thrombolysis or primary PCI, but perhaps a combination of pharmacological and mechanical reperfusion. Previous trials combining thrombolytics and PCI have been disappointing (Topol, TIMI IIa, Simoons), and the Maastricht trial and the PRAGUE-1 trial did not favour the combination of thrombolytic treatment plus transfer for PCI. However, modern PCI technique with the use of stents may change the setting and therefore new trials have been designed to test the possibility of combining thrombolytics with PCI.

Telemedicine: advantages in ST elevation MI

- Early warning to the hospital

- Urgent start of basic antithrombotic treatment

- Pre-hospital thrombolysis

- Direct referral to primary PCI 
The PACT trial showed preservation of myocardial function with alteplase preceding $\mathrm{PCI},{ }^{18}$ and this concept is being tested further in the ongoing ASCENT-4 trial.

The combination of reduced dose thrombolytics and glycoprotein IIb/IIIa blockers to facilitate PCI is currently being tested in the FINESSE trial. In the recently published BRAVE trial, ${ }^{19} 253$ patients were randomised to half dose reteplase plus abciximab or abciximab alone before primary PCI. In this trial where final infarct size was used as an end point, no difference between the two treatment groups could be demonstrated.

\section{CONCLUSION}

In countries where patients with acute ST elevation MI and symptoms of $<12$ hours duration can be transferred safely within two hours after diagnosis to a tertiary centre with a 24 hour PCI service, a treatment strategy of aspirin, clopidogrel, $\beta$ blockers, and abciximab, and quick reperfusion therapy with primary PCI including stenting can be recommended. This strategy is superior to conventional in-hospital thrombolysis. Pre-hospital thrombolysis (particularly in patients with symptoms of $<2$ hours duration) and facilitated PCI appears to represent another promising treatment option and should be compared to primary PCI in future trials.

\section{..................}

\section{Authors' affiliations}

S D Kristensen, H R Andersen, L Thuesen, L R Krusell, H E Bøtker, J F Lassen, T T Nielsen, Department of Cardiology, Skejby Hospital, Aarhus University Hospital, Aarhus N, Denmark

\section{REFERENCES}

1 Boersma E, Mercado N, Poldermans D, et al. Acute myocardial infarction. Lancet 2003;361:847-58.

- Excellent recent review on epidemiology, diagnosis, and treatment of acute myocardial infarction.

2 Van de Werf F, Ardissino D, Betriu A, et al. Management of acute myocardial infarction in patients presenting with ST-segment elevation. Eur Heart $J$ 2003;24:28-66.

- The current European Society of Cardiology guidelines, accepted for publication in August 2002.

3 Keeley EC, Boura JA, Grines CL. Primary angioplasty versus intravenous thrombolytic therapy for acute myocardial infarction: a quantitative review of 23 randomised trials. Lancet 2003;361:13-20.

- Recent meta-analysis conducted on all randomised trials comparing primary $\mathrm{PCl}$ to thrombolysis and also a separate meta-analysis on the transfer trials.

4 Vermeer F, Oude Ophuis AJM, van den Berg EJ, et al. Prospective randomized comparison between thrombolysis, rescue PTCA, and primary PTCA in patients with extensive myocardial infarction admitted to a hospital without PTCA facilities: a safety and feasibility study. Heart 1999;82:426-31

5 Widimsky P, Groch L, Zelizko M, et al. Multicentre randomized trial comparing transport to primary angioplasty vs immediate thrombolysis vs combined strategy for patients with acute myocardial infarction presenting to a community hospital without a catheterization laboratory. The PRAGUE study. Eur Heart J 2000;21:823-31.
6 Grines $\mathrm{CL}$, Westerhausen DR Jr, Grines LL, et al. A randomized trial of transfer for primary angioplasty versus on-site thrombolysis in patients with high-risk myocardial infarction: the air primary angioplasty in myocardial infarction study. J Am Coll Cardiol 2002;39:1713-9.

7 Widimsky $P$, Budesinsky $T$, Vorac $D$, et al. Long distance transport for primary angioplasty vs immediate thrombolysis in acute myocardial infarction. Eur Heart J 2003;24:94-104

- Second largest single study on streptokinase versus transferral for primary $\mathrm{PCl}$, showing a trend towards decreased mortality in those patients transferred for primary $\mathrm{PCl}$.

8 Andersen HR, Nielsen TT, Rasmussen K, et al. A comparison of coronary angioplasty with fibrinolytic therapy in acute myocardial infarction. N Engl J Med 2003;349:733-42.

- The largest and most recent study on alteplase versus primary $\mathrm{PCl}_{\text {, }}$ including a separate study showing that transferral to a tertiary centre for primary $\mathrm{PCl}$ is superior to thrombolysis given in the local hospital.

9 Andersen HR, Nielsen TT, Vesterlund T, et al. The Danish multicenter randomized study on fibrinolytic therapy versus acute coronary angioplasty in acute myocardial infarction: rationale and design of the DANAMI-2 trial. Am Heart J 2003;146:234-41

10 Dalby $M$, Bouzamando $A$, Lechat $P$, et al. Transfer for primary angioplasty versus immediate thrombolysis in acute myocardial infarction. Circulation 2003;108:1809-14.

Recent meta-analysis of the five transferral trials (references 4-8 in this paper) and the CAPTIM trial.

11 Bonnefoy E, Lapostolle F, Leizorovicz A, et al. Primary angioplasty versus prehospital fibrinolysis in acute myocardial infarction: a randomised study. Lancet 2002;360:825-29.

- The first publication on the French CAPTIM study showing a nonsignificant trend towards a better outcome in the primary $\mathrm{PCl}$ group compared to pre-hospital alteplase.

12 Hochman JS, Sleeper LA, Webb JG, et al. Early revascularization in acute myocardial infarction complicated by cardiogenic shock. SHOCK investigators: should we emergently revascularize occluded coronaries for cardiogenic shock, N Engl J Med 1999;341:625-34

- Randomised trial showing that patients aged $<75$ years with cardiogenic shock benefit from an invasive strategy with $\mathrm{PCl}$ or coronary artery bypass graft surgery.

13 Zijlstra F, Patel A, Jones M, et al. Clinical characteristics and outcome in patients with early $(<2 \mathrm{~h})$, intermediate $(2-4 \mathrm{~h})$ and late $(>4 \mathrm{~h})$ presentation treated by primary coronary angioplasty or thrombolytic therapy for acute myocardial infarction. Eur Heart J 2002:23:550-7.

14 Cannon CP, Gibson CM, Lambrew CT, et al. Relationship of symptom-onsetto-balloon time and door-to-balloon time with mortality in patients undergoing angioplasty for acute myocardial infarction. JAMA 2000;283:2941-7.

15 Morrison LJ, Verbeek PR, McDonald AC, et al. Mortality and prehospital thrombolysis for acute myocardial infarction. JAMA 2000;283:2686-92.

- Meta-analysis suggesting that pre-hospital thrombolyis decreases time to thrombolysis and all cause mortality compared to in-hospital thrombolysis.

16 Steg PG, Bonnefoy E, Chabaud S, et al. Impact of time to treatment on mortality after prehospital fibrinolysis or primary angioplasty. Circulation 2003; 108:2851-6.

- This paper reports the results of subgroup analysis of the CAPTIM study-a trend towards a better outcome for pre-hospital thrombolysis compared to transferral for primary $\mathrm{PCl}$ was found in patients with a duration of symptoms $<\mathbf{2}$ hours.

17 Topol EJ, Neumann FJ, Montalescot G. A preferred reperfusion strategy for acute myocardial infarction. J Am Coll Cardiol 2003;42:1886-9.

- Review and meta-analysis suggesting that the use of the platelet glycoprotein Ilb/Illa inhibitor abciximab improves outcome in patients undergoing primary $\mathrm{PCl}$.

18 Ross AM, Coyne KS, Reiner JS, et al. A randomized trial comparing primary angioplasty with a strategy of short-acting thrombolysis and immediate planned rescue angioplasty in acute myocardial infarction: the PACT trial. PACT investigators. Plasminogen-activator angioplasty compatibility trial. J Am Coll Cardiol 1999;34:1954-62.

19 Kastrati A, Mehilli J, Schotterbeck K, et al. Early administration of reteplase plus abcixiab vs abciximab alone in patients with acute myocardial infarction referrel for percutaneous coronary interveltion. JAMA 2004;291:947-54. 